\title{
Tracking the Secondary Photodynamics of the Green/Red Cyanobacteriochrome RcaE from Fremyella diplosiphon
}

\author{
Che-Wei Chang, ${ }^{1}$ Sean M. Gottlieb, ${ }^{1}$ Nathan C. Rockwell, ${ }^{2}$ Shelley S. Martin, ${ }^{2}$ \\ J. Clark Lagarias, ${ }^{2}$ and Delmar S. Larsen ${ }^{1 *}$ \\ ${ }^{1}$ Department of Chemistry and ${ }^{2}$ Department of Molecular and Cell Biology \\ One Shields Ave, University of California, Davis, 95616
}

*Corresponding Author: dlarsen@ucdavis.edu

\begin{abstract}
(100 words)
Cyanobacteriochrome RcaE regulates Type III complementary chromatic adaption in the cyanobacterium Fremyella diplosiphon by photoswitching between a green-absorbing dark state $\left({ }^{15 \mathrm{Z}_{\mathrm{g}}}\right)$ and red-absorbing photoproduct $\left({ }^{15 \mathrm{E}} \mathrm{P}_{\mathrm{r}}\right)$. Ultrafast photodynamics of RcaE involve tautomerization of the bilin chromophore, inhomogeneity, and the generation of three primary photointermediates in the forward reaction (Lumi- $G_{o}$, Lumi- $G_{r}$, and Lumi- $G_{f}$ ). The secondary photodynamics reported here show that only Lumi-Go evolves to ${ }^{15 E} \mathrm{Pr}_{\mathrm{r}}$ via spectrally similar Meta- $\mathrm{G}_{01}$ and Meta- $\mathrm{G}_{\mathrm{o}}$ intermediates, with a protonation reaction occurring at the final step on the millisecond timescale. Reverse reaction dynamics were characterized and reveal an unusually long-lived Lumi- $\mathrm{R}_{\mathrm{f}}$ photoproduct and a blue-shifted Meta- $\mathrm{R}_{\mathrm{y}}$ intermediate.
\end{abstract}




\section{INTRODUCTION}

Photoreceptor proteins allow organisms to respond to changing ambient light conditions via photobiological responses such as phototaxis, ${ }^{1,2}$ optimization of bacterial photosynthesis, ${ }^{3}$ and the regulation of gene expression. ${ }^{4-7}$ Multiple classes of photoreceptors have been characterized with spectral sensitivities ranging from the ultraviolet to the near-infrared, with longer wavelength sensitivity often associated with the bilin-binding phytochrome ${ }^{5,7,8}$ and cyanobacteriochrome (CBCR) families. ${ }^{7}$ 9-11 While phytochromes are typically associated with red and far-red responses, CBCRs span the entire visible spectrum and near ultraviolet. ${ }^{11,12}$ The photoresponses of both phytochromes and CBCRs are initiated by same the photoisomerization of the 15,16-double bond of the covalently attached bilin chromophore. However, the biophysical principles coupling this isomerization to the subsequent long-time dynamics necessary to generate photoresponses are still poorly understood. Such dynamics are a topic of great interest in understanding the mechanisms underlying protein photoactivity, and transient spectroscopies are optimal for such studies.

RcaE was genetically identified as a regulator of complementary chromatic acclimation in the filamentous cyanobacterium Fremyella diplosiphon and was subsequently shown to exhibit a green/red photocycle in agreement with the known action spectrum for CCA. ${ }^{13,14}$ RcaE photoswitches between a green-absorbing dark-adapted ${ }^{15 Z} \mathrm{Pg}$ state and a red-absorbing lightadapted photoproduct ${ }^{15 E} \mathrm{P}_{\mathrm{r}}$ state (Figure 1). The primary forward $\left({ }^{15 Z} \mathrm{P}_{\mathrm{g}} \rightarrow{ }^{15 E} \mathrm{P}_{\mathrm{r}}\right.$ ) and reverse $\left({ }^{15 E} \mathrm{P}_{\mathrm{r}} \rightarrow{ }^{15 \mathrm{Z}} \mathrm{P}\right)$ photoisomerization dynamics initiated after excitation of the phycocyanobilin (PCB) chromophore have previously been characterized with dispersed femtosecond pumpprobe (PP) and pump-dump-probe (PDP) spectroscopies. ${ }^{15} 16$ These transient studies resolved complex dynamics involving the interplay of long-living excited-states, multiple transient 
populations, excited-state tautomerization, photoisomerization, multilayered inhomogeneity, and reactive intermediates. ${ }^{16}$

In the primary (100 fs- $10 \mathrm{~ns}$ ) photodynamics of most phytochromes ${ }^{17-22}$ and CBCRs ${ }^{22-28}$ characterized to date, a single primary intermediate is typically resolved after the photoexcitation of the respective dark-adapted states. However, forward reaction photodynamics of RcaE are unique, with three primary intermediates observed after excitation of ${ }^{15 Z} \mathrm{P}_{\mathrm{g}}$ : Lumi-G $\mathrm{L}_{\mathrm{o}}$, Lumi-G and Lumi-G.. These primary photoproducts absorb in the orange $(595 \mathrm{~nm})$, red $(630 \mathrm{~nm})$, and far-red $(675 \mathrm{~nm})$, respectively. ${ }^{15,17}$ Upon initiation with green light, all three photoproducts are generated with characteristic yields. However, multipulse application of green and red light changes these yields, with Lumi-G $\mathrm{G}_{\mathrm{f}}$ increasing, Lumi-G $\mathrm{G}_{\mathrm{o}}$ decreasing, and Lumi-G $\mathrm{G}_{\mathrm{r}}$ remaining unaffected. ${ }^{16}$

Of these three coevolving primary photoproducts, the Lumi- $\mathrm{G}_{\mathrm{r}}$ intermediate is metastable and decays on a $900-p s$ timescale back into ${ }^{15 Z} \mathrm{P}_{\mathrm{g}}$ without evolving into a secondary intermediate. ${ }^{16}$ In contrast, both Lumi-Go and Lumi- $\mathrm{G}_{\mathrm{f}}$ intermediates persist beyond the 8-ns temporal window of the ultrafast experiments. Depending on the spectral properties of the incident light and the secondary dynamics $(>10 \mathrm{~ns})$ coupling these primary dynamics to the terminal light-adapted ${ }^{15 \mathrm{E}} \mathrm{P}_{\mathrm{r}}$ photoproduct state, the RcaE photoreceptor domain may be able to act in vivo either as a fluorescent probe or as a photoactive material. Optimizing this dualmodality potential in RcaE requires knowledge of how the primary intermediates propagate on longer timescales to generate the terminal ${ }^{15 E} \mathrm{Pr}_{\mathrm{r}}$ state. It is unclear if Lumi-G and Lumi-Go propagate independently to generate ${ }^{15} \mathrm{E}_{\mathrm{r}}$, or if they merge into a single pathway of sequentially evolving populations beyond the $>10 \mathrm{~ns}$ window. It is also possible that one of the intermediates may initiate a "dead end" pathway that terminates like Lumi- $\mathrm{G}_{\mathrm{r}}$ before regenerating ${ }^{15 \mathrm{Z}} \mathrm{P}_{\mathrm{g}}$. 
We here address these questions by characterizing the secondary photodynamics (10 ns $10 \mathrm{~ms}$ ) of RcaE with broadband transient absorption spectroscopy. While the primary dynamics of over 15 CBCRs have been resolved to date, ${ }^{15,16,22,23,25-27}$ the secondary dynamics (10 ns - 10 ms) of CBCRs have attracted less attention, with only four so characterized: AnPixJg2 from Nostoc sp. strain PCC 7120, ${ }^{29,} 30$ Slr1393g3 from Synechocystis sp. strain PCC6803, ${ }^{31}$ Tlr0924 from Thermosynechococcus elongatus ${ }^{24,32}$ and $\mathrm{NpF} 2164 \mathrm{~g} 3$ from Nostoc punctiforme. ${ }^{25}$ Our characterization of RcaE not only expands this list but also demonstrated that CBCRs exhibit markedly diverse photodynamics.

\section{MATERIALS AND METHODS}

2.1 Sample Preparation. The GAF domain of RcaE was purified from PCB-producing Escherichia coli cells ${ }^{13,33}$ as described previously. ${ }^{14}$ Static absorption spectra were acquired on a Cary 50 spectrophotometer modified to allow top-down illumination of the sample to initiate photochemistry with band-pass filters at $400 \pm 35 \mathrm{~nm}$ and $600 \pm 20 \mathrm{~nm}$.

2.2 Transient Signals. The instrumental setup for measuring secondary dynamics uses a design similar to that used to characterize the primary dynamics. ${ }^{25,}{ }^{34}$ Broadband probe pulses were generated by focusing a portion $(\sim 1 \mu \mathrm{J})$ of a 800 -nm Ti:Sapphire amplified laser (SpectraPhysics, Spitfire Pro) into a slowly translating $10-\mathrm{mm}$ thick $\mathrm{CaF}_{2}$ crystal. The probe pulses were imaged via a spectrograph (Oriel Instruments 77480) onto a 256-pixel InGaAs diode array (Hamamatsu C8061-01) after passing through the sample. The 526-nm excitation pulses for initiating secondary signals originate from the second harmonic output of an independent Qswitched diode-pumped solid-state Nd:YAG laser (Pulselas-A-532-300). The instrument response function was estimated at $\sim 550$ ps based on the rise times of the data. 
For forward reaction dynamics $\left({ }^{15 \mathrm{Z}} \mathrm{P}_{\mathrm{g}} \rightarrow{ }^{15 E} \mathrm{P} \mathrm{P}\right)$, the RcaE sample was flowed through a 1 mm thick optical cell ( $20 \mathrm{ml} / \mathrm{min})$ and was continuously illuminated with a $650-\mathrm{nm}$ LED to maintain the sample in the ${ }^{15 Z} \mathrm{Pg}_{\mathrm{g}}$ state. For the reverse reaction, the RcaE sample was illuminated with a 532-nm CW laser to photoconvert ${ }^{15 Z} \mathrm{Pg}$ to the ${ }^{15 E} \mathrm{P}_{\mathrm{r}}$ state. The $532-\mathrm{nm}$ excitation pulses used to initiate the secondary dynamics in this study are weakly resonant with the ${ }^{15 Z} \mathrm{P}_{\mathrm{r}}$ absorption spectrum and strongly resonant with the ${ }^{15 E} \mathrm{P}_{\mathrm{g}}$ spectrum (Figures 1 and $\mathrm{S} 1$, green curves). Consequently, the measured raw signals consist of both forward $\left({ }^{15 Z} \mathrm{P}_{\mathrm{g}} \rightarrow{ }^{15 E} \mathrm{P}_{\mathrm{r}}\right)$ and reverse $\left({ }^{15 E} \mathrm{P}_{\mathrm{r}} \rightarrow{ }^{15 \mathrm{Z}} \mathrm{Pg}\right)$ reaction signals. To extract the uncontaminated reverse reaction signals, "clean" forward reaction signals were first collected under continuous 650-nm laser diode illumination and were then subtracted from the raw reaction signals to clean reverse reaction signals with no observed bleach nor photoproduct bands from the forward reaction signals.

2.3 Multi-compartmental Global Analysis. The broadband transient signals were analyzed with the multicomponent global analysis formalism that has been successfully applied to characterizing the photodynamics of other CBCR systems. ${ }^{16,22,23,26-28,35-37}$ The complete methodology of this approach is described elsewhere, ${ }^{38}$ but the gist involves decomposing the transient data into a set of distinct populations with time-independent spectra and time-dependent amplitudes. For the data discussed here, the constituent populations are assumed to evolve via activated barrier-crossing dynamics, which can be described by first-order kinetics:

$$
\frac{\mathrm{d} n_{i}}{\mathrm{~d} t}=A_{i} I(t)+\sum_{j} K_{i j} n_{j}
$$


where the $n_{i}, A_{i} I(t)$, and $K_{i j}$ respectively represent the population of interest, the pump pulse profile, and the rate constant matrix elements that describes the connectivity of populations via the time constants of evolution of one population into another.

Global analysis models can differ in connectivity and thus in the interpretation of the extracted spectra and kinetics of the constituent populations (species): sequential, parallel, or an admixture of the two. For a sequential model $(\mathrm{A} \rightarrow \mathrm{B} \rightarrow \ldots)$, the resulting spectra are called Evolution-Associated Difference Spectra (EADS); for the parallel model $\left(\mathrm{A}^{*} \rightarrow \mathrm{A}, \mathrm{B}^{*} \rightarrow \mathrm{B}\right.$, $C^{*} \rightarrow C, \ldots$ ), the extracted spectra are called Decay Associated Difference Spectra (DADS) or Lifetime Associated Difference Spectra (LADS). If a model accurately describes the underlying photodynamics, then the extracted spectra are also Species Associated Difference Spectra (SADS) as they represent the "true" spectra of the constituent species. If the model does not accurately represent the underlying dynamics, then the extracted spectra are not SADS (although they can often be decomposed into a linear combination of SADS). While the analysis of the primary dynamics of RcaE required a more sophisticated target model,,${ }^{16}$ the secondary data here were initially analyzed within a sequential model (i.e., an EADS approach) before applying a more complex target model to extract underlying population dynamics.

\section{RESULTS}

$\mathrm{RcaE}$ is a member of the green/red family of CBCRs with a dark-adapted ${ }^{15 Z} \mathrm{Pg}$ state containing a deprotonated bilin $\pi$ system (Figure 1A). ${ }^{16}$ The ${ }^{15 \mathrm{Z}} \mathrm{Pg}$ absorption spectrum (green curve) peaks in the green region with a non-Gaussian spectral lineshape that is attributed to two tautomers of the PCB chromophore with either the B- or C-ring deprotonated ${ }^{39}$ that coexist in equilibrium. ${ }^{16}$ In contrast, the PCB chromophore in the ${ }^{15 \mathrm{E}} \mathrm{P}_{\mathrm{r}}$ state is fully protonated with a nonGaussian absorption spectrum (red curve) peaking at $660 \mathrm{~nm}$ with a high-energy shoulder. 
The forward reaction transient spectra of RcaE at select probe times are contrasted in Figure 2A. Since the 550-ps instrument response is appreciably slower than the excited-state ${ }^{15 Z} \mathrm{P}_{\mathrm{g}} *$ kinetics, ${ }^{15}, 16$ the measured spectra primarily track the subsequent ground-state intermediates. These spectra are the combination of a negative absorption from a ground-state bleach (GSB, 450-560 nm) due to loss of the ${ }^{15 Z} \mathrm{P}_{\mathrm{g}}$ ground-state population and positive absorption from new photoproduct bands $(560-710 \mathrm{~nm})$. The $2 \mathrm{~ns}$ spectrum (black curve) peaks at $593 \mathrm{~nm}$ with a long wavelength tail ${ }^{15,16}$ and consists of the Lumi-Go ( 595 nm), Lumi-Gr $(\sim 630 \mathrm{~nm})$, and Lumi-G $(\sim 700 \mathrm{~nm})$ primary photoproducts. This spectrum then evolves into a blue-shifted spectrum peaking at $\sim 575 \mathrm{~nm}$ with a substantial loss of amplitude of the red portion of the spectrum (10 ns spectrum; red curve); this evolution is primarily ascribed to the decay of Lumi- $\mathrm{G}_{\mathrm{r} .}{ }^{16}$ However, the broad far-red absorption attributed to the Lumi- $\mathrm{G}_{\mathrm{f}}$ intermediate at $\sim 700$ $\mathrm{nm}$ still persists. The subsequent $100 \mathrm{~ns}-100 \mu$ s spectra shift slightly in peak wavelength and amplitude (Figure 2A; inset) before eventually evolving into ${ }^{15 \mathrm{E}} \mathrm{P}_{\mathrm{r}}(1 \mathrm{~ms}$; brown curve). While the kinetics traces probing the ${ }^{15 \mathrm{Z}} \mathrm{Pg}$ bleach, Lumi-Go, and ${ }^{15 \mathrm{E}} \mathrm{P}_{\mathrm{r}}$ populations (Figure $2 \mathrm{~B}$ and $2 \mathrm{C}$ ) exhibit comparable dynamics, the Lumi-G population (Figure 2D) $15,1615,1615,1615,1615,1615$, $1615-$ 16 15-16 15-16 15-16 15-16 16,45,44 exhibits a unique $\sim 5$ s decay timescale, indicating it independently evolves as implied by previous interpretations of the inhomogeneous primary dynamics..$^{15,16}$

The data were analyzed within a sequential model (Figure 3) that required six compartments to capture the full observed dynamics. In contrast to the secondary dynamics of other CBCRs, ${ }^{25,29-31,40}$ which exhibit significant spectral evolution, the extracted EADS exhibit only small changes of amplitudes and peak wavelengths, with the exception of the initial excitedstate ${ }^{15 Z} \mathrm{P}_{\mathrm{g}} *$ and terminal ${ }^{15 \mathrm{E}} \mathrm{P}_{\mathrm{r}}$ spectra (EADS1 and EADS6). Based on this EADS analysis, the previous PP signals, and the interpretation of the raw data in Figure 2, a more complex 
inhomogeneous target model was adopted to analyze the photodynamics (Figure 4). The spectra of three primary photoproducts (Lumi-Go, Lumi- $\mathrm{G}_{\mathrm{r}}$, and Lumi-G $\mathrm{G}_{\mathrm{f}}$ ) were extracted with decay lifetimes of $28 \mathrm{~ns}, 900 \mathrm{ps}$, and $5 \mu \mathrm{s}$, respectively. These results agree well with the modeled interpretation of the primary signals. ${ }^{16}$ While Lumi-Go peaks near $595 \mathrm{~nm}$ with a narrow peak, both Lumi- $\mathrm{G}_{\mathrm{r}}$ and Lumi-G have broader absorption bands peaking near $625 \mathrm{~nm}$ and $675 \mathrm{~nm}$, respectively. Lumi-Go generates a secondary photoproduct population (Meta-Go1) that peaks near $592 \mathrm{~nm}$ and evolves into Meta- $\mathrm{G}_{\mathrm{o} 2}$ on a $500 \mu$ s timescale. Meta- $\mathrm{G}_{\mathrm{o} 2}$ in turn evolves into ${ }^{15 E} \mathrm{Pr}$ on a $2.3 \mathrm{~ms}$ timescale (Figure 4B; unfilled circles). The uncertainty of the 2.3 timeconstant ( $\pm 400 \mu$ s ) is greater than the $\pm 10 \%$ typical of the analysis since less than decay over half the timeconstant was resolved in the data.

The reverse reaction photodynamics of RcaE (Figure 5) are simpler than the forward dynamics. Photoexcitation of ${ }^{15 \mathrm{E}} \mathrm{P}_{\mathrm{r}}$ generates only a single Lumi-R $\mathrm{R}_{\mathrm{f}}$ photoproduct absorbing near $680 \mathrm{~nm}^{16}$ with kinetics that are similar to the excited-state dynamics of ${ }^{15 Z} \mathrm{P}_{\mathrm{r}} *$ in red/green CBCRs, ${ }^{26,27,41}$ although on a slower timescale ( $\left.\sim 380 \mathrm{ps}\right)$. Lumi-Rf persists with a lifetime of 110 $\mu$ s (Figure 6) before forming a Meta-Ry population that peaks near $580 \mathrm{~nm}$ but is poorly resolved. EADS1 represents formation of the ${ }^{15 Z} \mathrm{Lumi}-\mathrm{R}_{\mathrm{f}}$ photoproduct, with a ground-state bleach of ${ }^{15 E} \operatorname{P}_{\mathrm{r}}$ peaking at $640 \mathrm{~nm}$ and a positive absorption band near $685 \mathrm{~nm}$ (Figure 6). This photo-intermediate persists until $110 \mu \mathrm{s}$, before evolving into the secondary Meta- $\mathrm{R}_{\mathrm{y}}$ photoproduct peaking near $590 \mathrm{~nm}$ (EADS 2; Figures 5 and 6). Final formation of ${ }^{15 Z} \mathrm{Pg}$ was not resolved. A similar evolution into a blue-shifted intermediate is also observed in the forward photodynamics of other red/green CBCRs.

\section{Discussion}


RcaE exhibits the most complex primary and secondary dynamics of any CBCR resolved to date, conceptualized in the photocycle presented in Figure 7. The primary forward dynamics are strongly controlled by the inhomogeneity of the sample, ascribed in part to the two tautomers of the PCB chromophore. ${ }^{15,} 16,39$ Of the three primary Lumi photoproducts formed after excitation of ${ }^{15 Z} \mathrm{P}_{\mathrm{g}}$, only Lumi-G $\mathrm{G}_{\mathrm{o}}$ propagates forward to generate ${ }^{15 \mathrm{E}} \mathrm{P}_{\mathrm{r}}$ via two spectrally similar, but not identical, Meta- $\mathrm{G}_{01}$ and Meta- $\mathrm{G}_{02}$ intermediates. The Lumi- $\mathrm{G}_{\mathrm{o}}$ photointermediate generated in the forward dynamics of RcaE after excitation of ${ }^{15 Z} \mathrm{P}_{\mathrm{g}}$ qualitatively resembles the Lumi-Go primary intermediate of the reverse photodynamics of the red/green CBCRs NpR6012g4, AnPixJg2, and Slr1393g3, in which the chromophore in the 15E configuration in the green-absorbing state. ${ }^{23,26,28,42}$ However, the subsequent secondary dynamics of RcaE do not exhibit the strong spectral shifting observed in red/green CBCRs. This difference likely originates from the differing nature of the photodynamics, since the ${ }^{15 \mathrm{E}} \mathrm{P}_{\mathrm{g}} \rightarrow{ }^{15 \mathrm{Z}} \mathrm{P}_{\mathrm{r}}$ evolution in red/green CBCRs is primarily ascribed to structural evolution of PCB from a starting $15 \mathrm{E}$ geometry, ${ }^{43}$ while the ${ }^{15 Z} \mathrm{Pg}_{\mathrm{g}} \rightarrow{ }^{15 \mathrm{E}} \mathrm{P}_{\mathrm{r}}$ evolution in RcaE involves both structural evolution (from a starting $15 Z$ geometry) and a proton transfer reaction (Figure 7). Unfortunately, it is unclear from these visible data as which step the deprotonation event occurs, which likely requires vibrational sensitive experiments to resolve. A proposed kinetic isotope experiment with $\mathrm{D}_{2} \mathrm{O}$ exchange of the $\mathrm{H}_{2} \mathrm{O}$ buffer is planned to help address this question.

It is unclear at what stage the proton transfer occurs in the secondary signals in Figures 4 and 5 , but it does not occur rapidly in the reverse reaction (excitation of ${ }^{15}{ }^{2} \mathrm{Pr}$ ). Of the three CBCR systems whose secondary dynamics have been characterized, Lumi- $\mathrm{R}_{\mathrm{f}}$ formed in the reverse direction of RcaE exhibits the longest living primary photoproduct resolved to date (110 $\mu$ s decay constant). ${ }^{29-31}$ In contrast, decay of Lumi-R $R_{f}$ in AnPixJg2 (termed APr648) is appreciably 
faster $(<100 \mathrm{~ns})^{29}, 30,44$ and no clear evidence of Lumi- $\mathrm{R}_{\mathrm{f}}$ was resolved in the red/green Slr1393g3 CBCR (although the authors suspected that Lumi-Rf may be obscured by overlapping GSB and ESA bands). ${ }^{28,31} \mathrm{~A}$ molecular level description of the underlying three-order difference in lifetimes for AnPixJg2, Slr1393g3, and RcaE is lacking.

However, while the primary dynamics of AnPixJg2 have not been resolved, the average excited-state lifetime of ${ }^{15 Z} \mathrm{P}_{\mathrm{r}}{ }^{*}$ in $\mathrm{S} 1 \mathrm{r} 1393 \mathrm{~g} 3$ was recently reported by Wachtveitl and coworkers at $\sim 20 \mathrm{ps},{ }^{28}$ which is comparable to the lifetimes of many of the red/green CBCRs reported from Nostoc punctiforme. ${ }^{27}$ The 380 ps excited-state lifetime of ${ }^{15} \mathrm{P}_{\mathrm{r}}{ }^{*}$ in $\mathrm{RcaE}$ is comparable to the slower members of this set of these red/green CBCRs. While it is tempting to propose that mutual mechanism exists in controlling both excited-state lifetimes and subsequent primary intermediates decay times in CBCRs, establishing such a correlation requires additional secondary signals to be characterized and is a topic of further study.

\section{Concluding Comments}

Our work demonstrates that Lumi-Gr and Lumi-Gf, two of the three primary photoproducts formed in the forward photoreaction of RcaE, are nonproductive. This result implies that the observed ability to enhance Lumi- $\mathrm{G}_{\mathrm{f}}$ formation at the expense of Lumi- $\mathrm{G}_{\mathrm{o}}$ with multipulse PDP spectroscopy transfers the excited-state population from a productive pathway to a nonproductive one. RcaE thus may be a CBCR system that allows selective transfer from photoactive sub-populations to fluorescent ones via multipulse techniques, behavior with a range of potentially intriguing applications in optogenetic or synthetic biology approaches.

\section{ACKNOWLEDGEMENTS}


Dr. Mikas Vengris (Light Conversion Ltd.) is acknowledged for the donation of global and target analysis software package. The research reported in this publication was supported by the Biosciences Division, Office of Basic Energy Sciences, Office of Science, United States Department of Energy (DOE DE-FG02-09ER16117) to both J.C.L. and D.S.L. The content is solely the responsibility of the authors and does not necessarily represent the official views of the Department of Energy.

\section{REFERENCES}

[1] Campbell, E. L., Hagen, K. D., Chen, R., Risser, D. D., Ferreira, D. P., and Meeks, J. C. (2015) Genetic Analysis Reveals the Identity of the Photoreceptor for Phototaxis in Hormogonium Filaments of Nostoc punctiforme, J Bacteriol 197, 782-791.

[2] Armitage, J. P., and Hellingwerf, K. J. (2003) Light-induced behavioral responses ('phototaxis') in prokaryotes, Photosynth Res 76, 145-155.

[3] van der Horst, M. A., Laan, W., Yeremenko, S., Wende, A., Palm, P., Oesterhelt, D., and Hellingwerf, K. J. (2005) From primary photochemistry to biological function in the blue-light photoreceptors PYP and AppA, Photoch Photobio Sci 4, 688-693.

[4] Hoff, W. D., van der Horst, M. A., Nudel, C. B., and Hellingwerf, K. J. (2009) Prokaryotic phototaxis, Methods in Molecular Biology 571, 25-49.

[5] Franklin, K. A., and Quail, P. H. (2010) Phytochrome functions in Arabidopsis development, Journal of Experimental Botany 61, 11-24.

[6] Moglich, A., Yang, X. J., Ayers, R. A., and Moffat, K. (2010) Structure and Function of Plant Photoreceptors, Annual Review of Plant Biology, Vol 61 61, 21-47.

[7] Auldridge, M. E., and Forest, K. T. (2011) Bacterial phytochromes: More than meets the light, Critical Reviews in Biochemistry and Molecular Biology 46, 67-88.

[8] Rockwell, N. C., and Lagarias, J. C. (2010) A Brief History of Phytochromes, Chemphyschem 11, 11721180.

[9] Ishizuka, T., Kamiya, A., Suzuki, H., Narikawa, R., Noguchi, T., Kohchi, T., Inomata, K., and Ikeuchi, M. (2011) The Cyanobacteriochrome, TePixJ, Isomerizes Its Own Chromophore by Converting Phycocyanobilin to Phycoviolobilin, Biochemistry 50, 953-961.

[10] Rockwell, N. C., Martin, S. S., Feoktistova, K., and Lagarias, J. C. (2011) Diverse two-cysteine photocycles in phytochromes and cyanobacteriochromes, Proceedings of the National Academy of Sciences of the United States of America 108, 11854-11859.

[11] Rockwell, N. C., Martin, S. S., Gulevich, A. G., and Lagarias, J. C. (2012) Phycoviolobilin formation and spectral tuning in the DXCF cyanobacteriochrome subfamily, Biochemistry 51, 1449-1463.

[12] Rockwell, N. C., Martin, S. S., and Lagarias, J. C. (2012) Red/Green Cyanobacteriochromes: Sensors of Color and Power, Biochemistry 51, 9667-9677.

[13] Kehoe, D. M., and Grossman, A. R. (1996) Similarity of a chromatic adaptation sensor to phytochrome and ethylene receptors, Science 273, 1409-1412.

[14] Hirose, Y., Rockwell, N. C., Nishiyama, K., Narikawa, R., Ukaji, Y., Inomata, K., Lagarias, J. C., and Ikeuchi, M. (2013) Green/red cyanobacteriochromes regulate complementary chromatic 
acclimation via a protochromic photocycle, Proceedings of the National Academy of Sciences 110, 4974-4979.

[15] Sean M. Gottlieb, C.-W. C., Randeep J. Hayer, Nathan C. Rockwell, J. Clark Lagarias, Delmar S. Larsen. (2013) Conservation and Diversity in the Primary Forward Photodynamics of Red/Green Cyanobacteriochromes, Biochemistry 54, 1028-1042.

[16] Gottlieb, S. M., Chang, C. W., Martin, S. S., Rockwell, N. C., Lagarias, J. C., and Larsen, D. S. (2014) Optically Guided Photoactivity: Coordinating Tautomerization, Photoisomerization, Inhomogeneity, and Reactive Intermediates within the RcaE Cyanobacteriochrome, J Phys Chem Lett 5, 1527-1533.

[17] Andel, F., Hasson, K. C., Gai, F., Anfinrud, P. A., and Mathies, R. A. (1997) Femtosecond timeresolved spectroscopy of the primary photochemistry of phytochrome, Biospectroscopy 3, 421433.

[18] Toh, K. C., Stojkovic, E. A., Rupenyan, A. B., van Stokkum, I. H., Salumbides, M., Groot, M. L., Moffat, K., and Kennis, J. T. (2011) Primary reactions of bacteriophytochrome observed with ultrafast mid-infrared spectroscopy, The journal of physical chemistry. A 115, 3778-3786.

[19] Savikhin, S., Wells, T., Song, P. S., and Struve, W. S. (1993) Ultrafast Pump-Probe Spectroscopy of Native Etiolated Oat Phytochrome, Biochemistry 32, 7512-7518.

[20] Muller, M. G., Lindner, I., Martin, I., Gartner, W., and Holzwarth, A. R. (2008) Femtosecond kinetics of photoconversion of the higher plant photoreceptor phytochrome carrying native and modified chromophores, Biophys J 94, 4370-4382.

[21] Toh, K. C., Stojkovic, E. A., van Stokkum, I. H. M., Moffat, K., and Kennis, J. T. M. (2010) Protontransfer and hydrogen-bond interactions determine fluorescence quantum yield and photochemical efficiency of bacteriophytochrome, Proceedings of the National Academy of Sciences of the United States of America 107, 9170-9175.

[22] Kim, P. W., Pan, J., Rockwell, N. C., Chang, C. W., Taylor, K. C., Lagarias, J. C., and Larsen, D. S. (2012) Ultrafast E to Z photoisomerization dynamics of the Cph1 phytochrome, Chem Phys Lett 549, 8692.

[23] Freer, L. H., Kim, P. W., Corley, S. C., Rockwell, N. C., Zhao, L., Thibert, A. J., Lagarias, J. C., and Larsen, D. S. (2012) Chemical Inhomogeneity in the Ultrafast Dynamics of the DXCF Cyanobacteriochrome TIr0924, J Phys Chem B 116, 10571-10581.

[24] Hardman, S. J. O., Hauck, A. F. E., Clark, I. P., Heyes, D. J., and Scrutton, N. S. (2014) Comprehensive Analysis of the Green-to-Blue Photoconversion of Full-Length Cyanobacteriochrome TIr0924, Biophysical Journal 107, 2195-2203.

[25] Gottlieb, S. M., Kim, P. W., Corley, S. C., Madsen, D., Hanke, S. J., Chang, C. W., Rockwell, N. C., Martin, S. S., Lagarias, J. C., and Larsen, D. S. (2014) Primary and Secondary Photodynamics of the Violet/Orange Dual-Cysteine NpF2164g3 Cyanobacteriochrome Domain from Nostoc punctiforme, Biochemistry 53, 1029-1040.

[26] Chang, C. W., Gottlieb, S. M., Kim, P. W., Rockwell, N. C., Lagarias, J. C., and Larsen, D. S. (2013) Reactive ground-state pathways are not ubiquitous in red/green cyanobacteriochromes, J Phys Chem B 117, 11229-11238.

[27] Gottlieb, S. M., Kim, P. W., Chang, C. W., Hanke, S. J., Hayer, R. J., Rockwell, N. C., Martin, S. S., Lagarias, J. C., and Larsen, D. S. (2015) Conservation and diversity in the primary forward photodynamics of red/green cyanobacteriochromes, Biochemistry 54, 1028-1042.

[28] C Slavov, X Xu, K Zhao, W Gärtner, and Wachtveitl, J. (2015) Detailed insight into the ultrafast photoconversion of the cyanobacteriochrome SIr1393 from Synechocystis sp, Biochimica Et Biophysica Acta 1847. 
[29] Narikawa, R., Ishizuka, T., Ochiai, Y., Katayama, M., Kohchi, T., and Ikeuchi, M. (2007) Novel greenred photoreversible cyanobacteriochrome (AnPixJ): Is the chromophore of green-absorbing form identical to that of Pfr of phytochrome?, Plant Cell Physiol 48, S102-S102.

[30] Fukushima, Y., Iwaki, M., Narikawa, R., Ikeuchi, M., Tomita, Y., and Itoh, S. (2011) Photoconversion Mechanism of a Green/Red Photosensory Cyanobacteriochrome AnPixJ: Time-Resolved Optical Spectroscopy and FTIR Analysis of the AnPixJ-GAF2 Domain, Biochemistry 50, 6328-6339.

[31] Xu, X. L., Gutt, A., Mechelke, J., Raffelberg, S., Tang, K., Miao, D., Valle, L., Borsarelli, C. D., Zhao, K. H., and Gartner, W. (2014) Combined Mutagenesis and Kinetics Characterization of the BilinBinding GAF Domain of the Protein SIr1393 from the Cyanobacterium Synechocystis PCC6803, Chembiochem 15, 1190-1199.

[32] Hauck, A., Hardman, S., Kutta, R., Greetham, G., Heyes, D., and Scrutton, N. (2014) The photoinitiated reaction pathway of full-length cyanobacteriochrome TIr0924 monitored over 12 orders of magnitude., J Biol Chem 20, 17747-17757.

[33] Hirose, Y., Shimada, T., Narikawa, R., Katayama, M., and Ikeuchi, M. (2008) Cyanobacteriochrome $\mathrm{CcaS}$ is the green light receptor that induces the expression of phycobilisome linker protein, Proc Natl Acad Sci U S A 105, 9528-9533.

[34] Song, S. H., Madsen, D., van der Steen, J. B., Pullman, R., Freer, L. H., Hellingwerf, K. J., and Larsen, D. S. (2013) Primary Photochemistry of the Dark- and Light-Adapted States of the YtvA Protein from Bacillus subtilis, Biochemistry 52, 7951-7963.

[35] Kim, P. W., Rockwell, N. C., Freer, L. H., Chang, C. W., Martin, S. S., Lagarias, J. C., and Larsen, D. S. (2013) Unraveling the Primary Isomerization Dynamics in Cyanobacterial Phytochrome Cph1 with Multi-pulse Manipulations, J Phys Chem Lett 4, 2605-2609.

[36] Kim, P. W., Freer, L. H., Rockwell, N. C., Martin, S. S., Lagarias, J. C., and Larsen, D. S. (2011) SecondChance Initiation Dynamics of the Cyanobacterial Photocycle in the NpR6012 GAF 4 Domain of Nostoc Punctiforme, Journal of American Chemical Society accepted.

[37] Kim, P. W., Rockwell, N. C., Martin, S. S., Lagarias, J. C., and Larsen, D. S. (2014) Heterogeneous Photodynamics of the P-fr State in the Cyanobacteriai Phytochrome Cph1, Biochemistry 53, 4601-4611.

[38] van Stokkum, I. H. M., Larsen, D. S., and van Grondelle, R. (2004) Global and target analysis of timeresolved spectra, Biochimica Et Biophysica Acta-Bioenergetics 1657, 82-104.

[39] Gottlieb, S. M., Kim, P. W., Rockwell, N. C., Hirose, Y., Ikeuchi, M., Lagarias, J. C., and Larsen, D. S. (2013) Primary Photodynamics of the Green/Red-Absorbing Photoswitching Regulator of the Chromatic Adaptation E Domain from Fremyella diplosiphon, Biochemistry 52, 8198-8208.

[40] Gottlieb, S. M., Corley, S. C., Madsen, D., and Larsen, D. S. (2012) A flexible light emitting diodebased broadband transient-absorption spectrometer, Rev Sci Instrum 83.

[41] Kim, P. W., Freer, L. H., Rockwell, N. C., Martin, S. S., Lagarias, J. C., and Larsen, D. S. (2012) Femtosecond Photodynamics of the Red/Green Cyanobacteriochrome NpR6012g4 from Nostoc punctiforme. 1. Forward Dynamics, Biochemistry 51, 608-618.

[42] Escobar, F. V., Utesch, T., Narikawa, R., Ikeuchi, M., Mroginski, M. A., Gartner, W., and Hildebrandt, P. (2013) Photoconversion Mechanism of the Second GAF Domain of Cyanobacteriochrome AnPixJ and the Cofactor Structure of Its Green-Absorbing State, Biochemistry 52, 4871-4880.

[43] Rockwell, N. C., Martin, S. S., and Lagarias, J. C. (2012) Mechanistic Insight into the Photosensory Versatility of DXCF Cyanobacteriochromes, Biochemistry 51, 3576-3585.

[44] Narikawa, R., Fukushima, Y., Ishizuka, T., Itoh, S., and Ikeuchi, M. (2008) A novel photoactive GAF domain of cyanobacteriochrome AnPixJ that shows reversible green/red photoconversion, Journal of Molecular Biology 380, 844-855. 


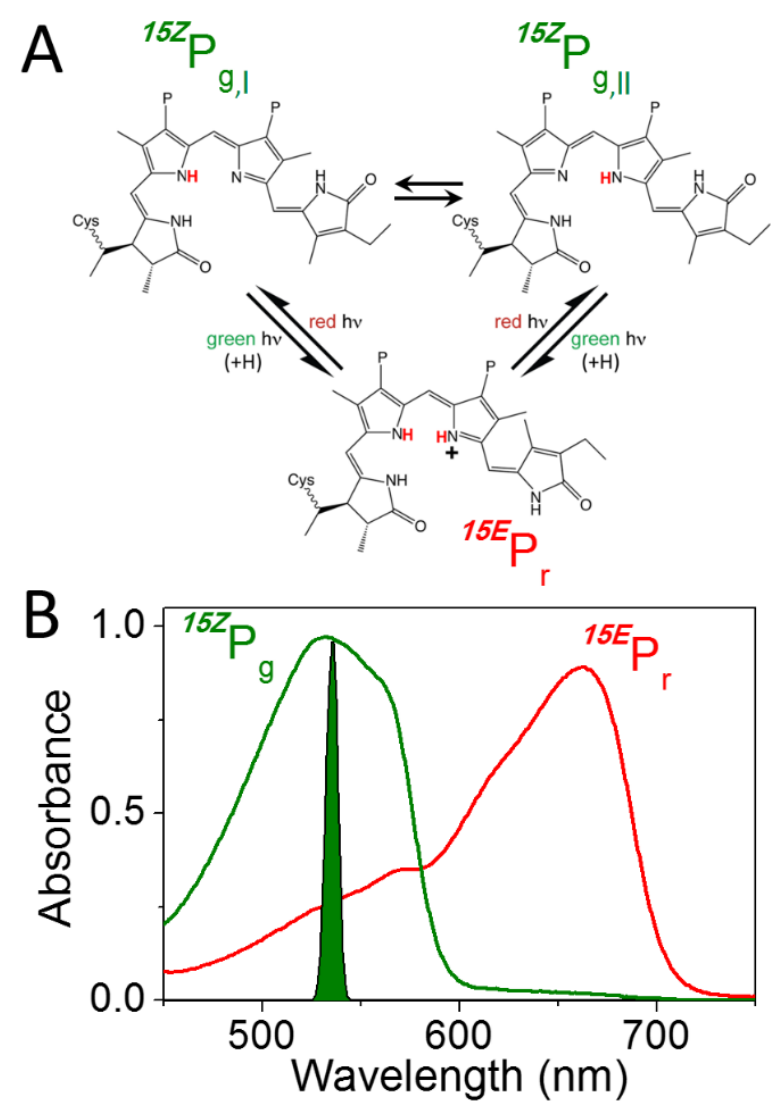

Figure 1: The photochromic photocycle of the domain from Fremyella diplosiphon (RcaE). In this photocycle, the phycocyanobilin (PCB) chromophore exhibits a deprotonated, greenabsorbing ${ }^{15 Z} \mathrm{Pg}$ dark state and a protonated, red-absorbing ${ }^{15 E} \mathrm{P}_{\mathrm{r}}$ photoproduct. Two tautomers exist for $\mathrm{PCB}$ in the ${ }^{15 \mathrm{Z}} \mathrm{Pg}$ state. (A) The molecular geometries and (B) static absorption spectra of RcaE in the ${ }^{15 Z} \mathrm{Pg}$ and ${ }^{15 E} \mathrm{P}_{\mathrm{r}}$ states. The excitation pulse spectrum (filled green curve) was used to initiate the reaction dynamics of both forward and reverse directions. 


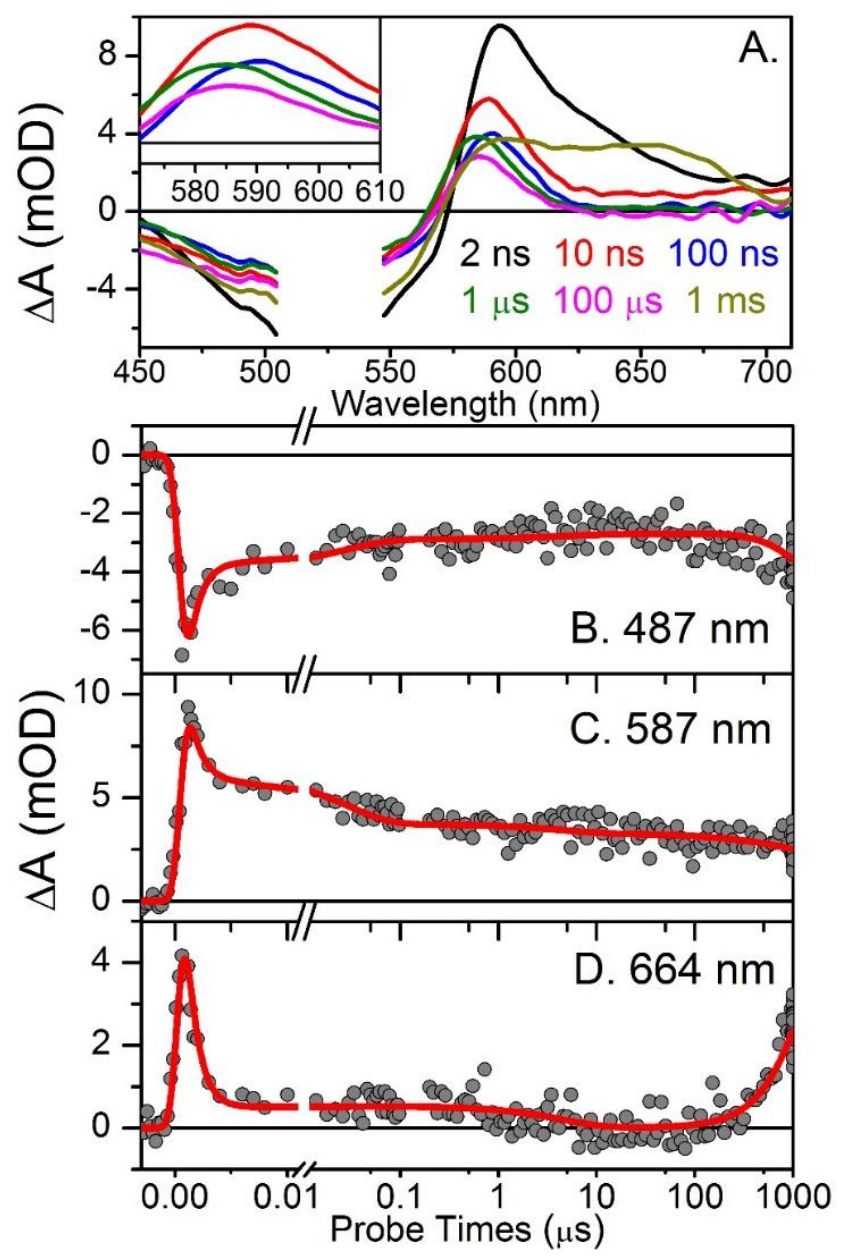

Figure 2. Transient spectra of the (A) forward dynamics $\left({ }^{15 Z} \mathrm{P}_{\mathrm{g}} \rightarrow{ }^{15 E} \mathrm{P}_{\mathrm{r}}\right)$ at selected probe times and (B-D) kinetic traces at selected wavelengths. The global analysis fits (red curves in B-D) are the result of the inhomogeneous target analysis model in Figure 4. 


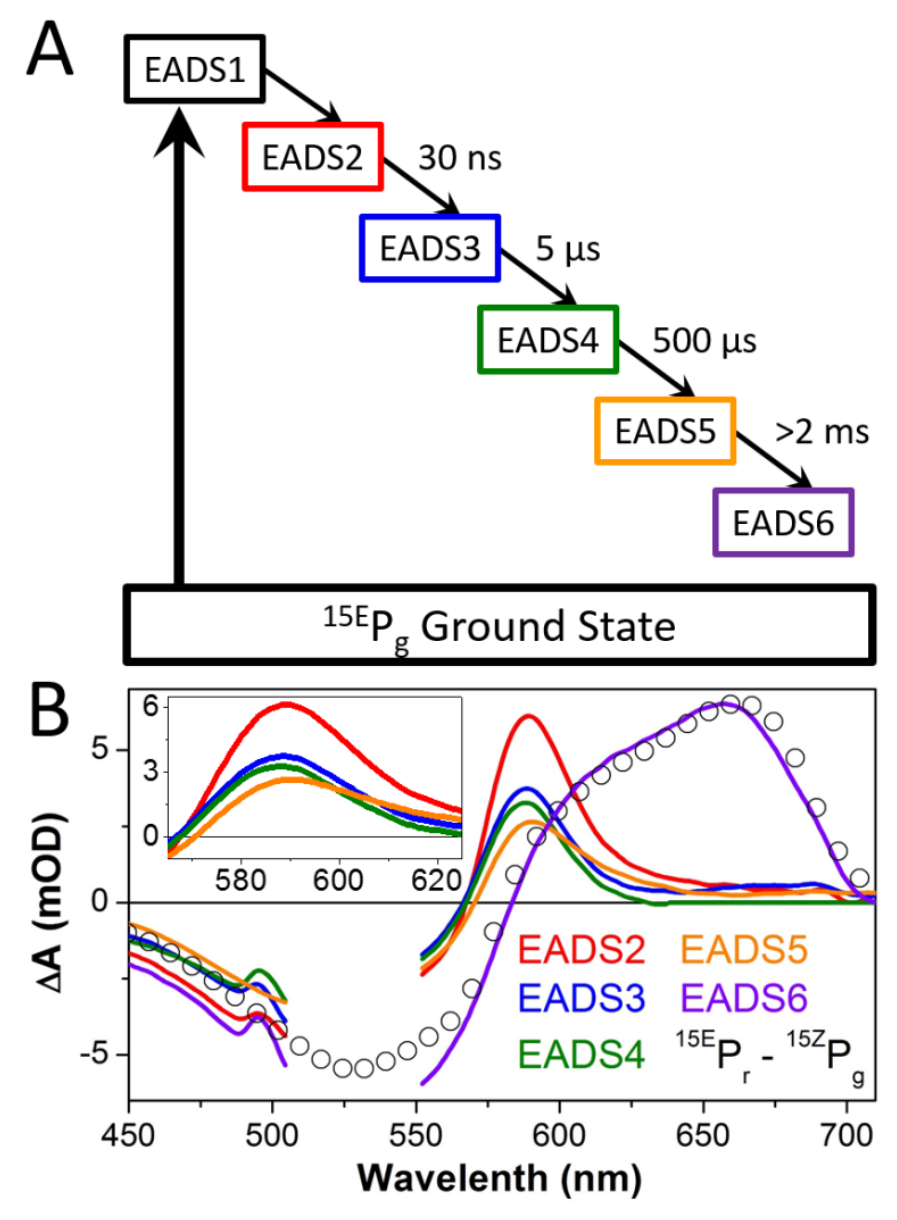

Figure 3. Sequential EADS analysis of the secondary forward $\left({ }^{15 E} \mathrm{P}_{\mathrm{g}} \rightarrow{ }^{15 Z} \mathrm{P}_{\mathrm{r}}\right)$ photodynamics. The first EADS (EADS1) is presented in Figure S1 to facilitate the comparison of ground-state photoproduct populations. EADS1 has a decaying lifetime $\sim 1 \mathrm{~ns}$ that is not poorly resolved due to the IRF. The ${ }^{15 \mathrm{E}} \mathrm{Pr}_{\mathrm{r}}-{ }^{15 \mathrm{Z}} \mathrm{Pg}$ difference spectra (unfilled circles in Figure $3 \mathrm{~B}$ ) is shown as a visual aid. 


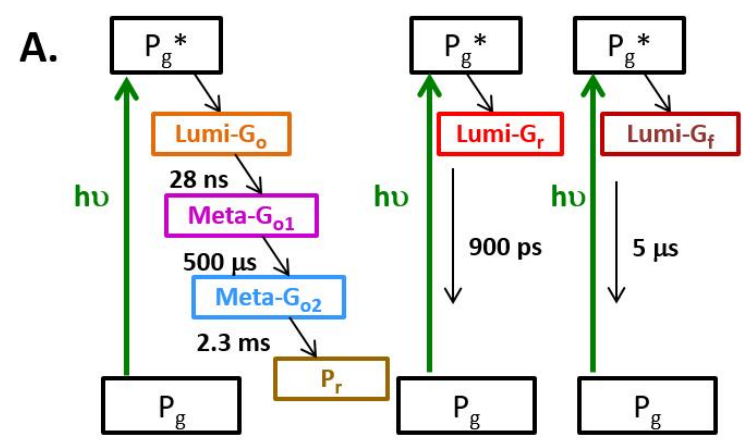

B.

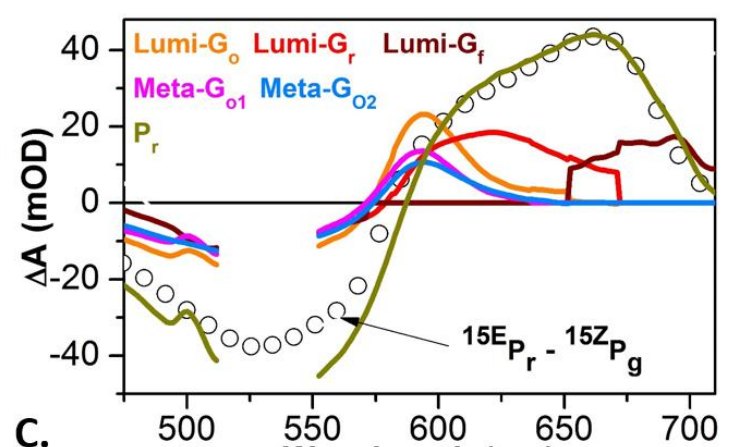

C.

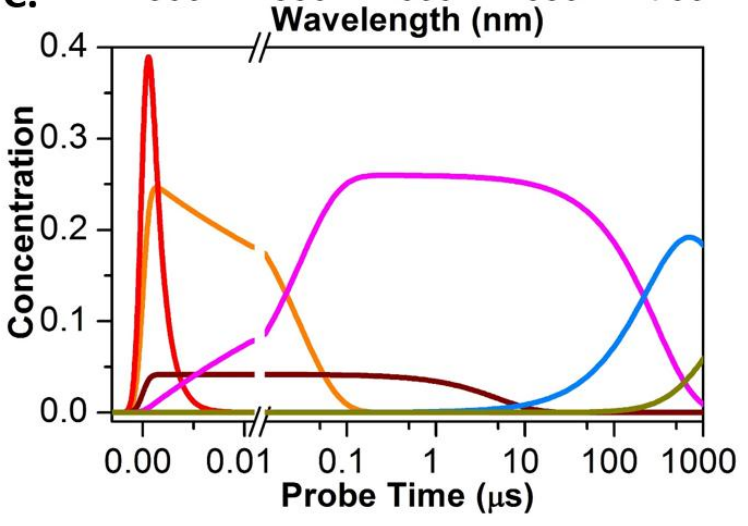

Figure 4. The inhomogeneous target model analysis for the secondary forward $\left({ }^{15 Z} \mathrm{P}_{\mathrm{g}} \rightarrow{ }^{15 E} \mathrm{P}_{\mathrm{r}}\right)$ dynamics: (A) the target model, (B) SADS, and (C) the concentration profiles for the corresponding compartments. The ${ }^{15} \mathrm{E}_{\mathrm{r}}-{ }^{15 \mathrm{Z}} \mathrm{P}$ difference spectra (unfilled circles) is shown for comparison. The excited-state ${ }^{15 Z} \mathrm{Pg}_{\mathrm{g}} * \mathrm{SADS}$ is poorly resolved due to the 550-ps IRF and not shown (Figure S2). 


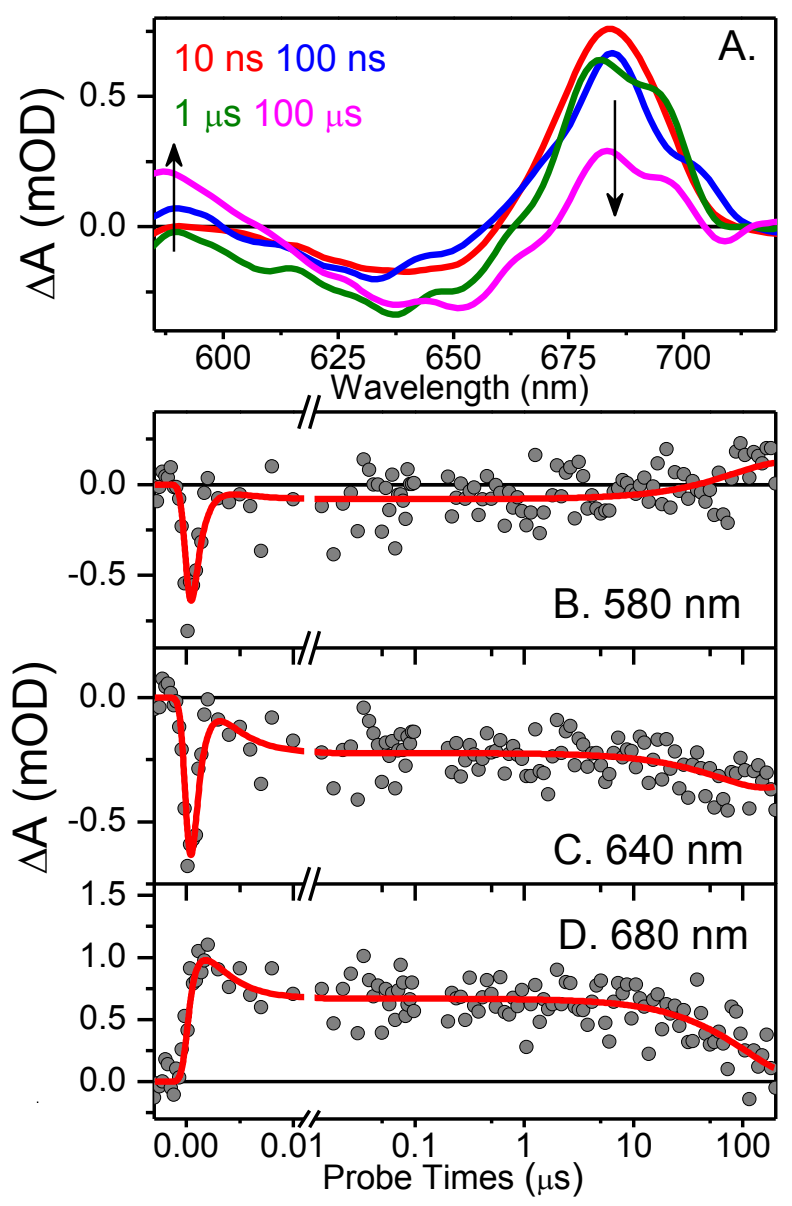

Figure 5. (A) Transient spectra of the secondary reverse photodynamics $\left({ }^{15 E} \mathrm{P}_{\mathrm{r}} \rightarrow{ }^{I Z E} \mathrm{Pg}\right)$ at selected probe times and (B-D) PP kinetic traces at selected wavelengths. The global analysis fits (red curves) results from the two-state sequential model in Figure 6A. 


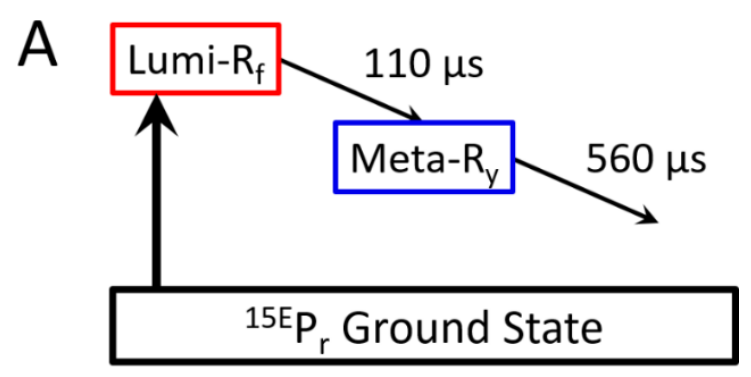

B

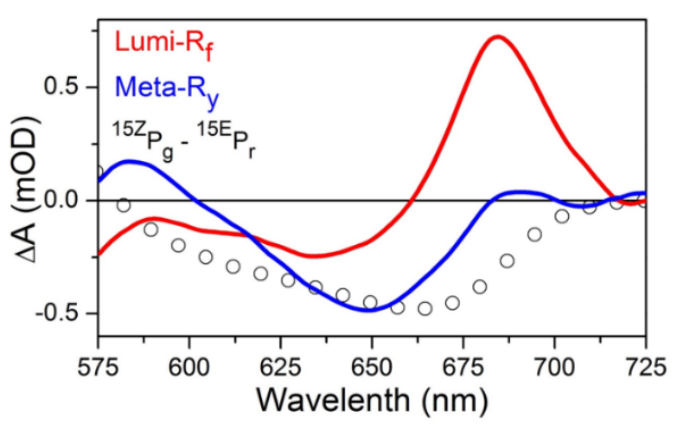

Figure 6. Sequential analysis of the secondary reverse photodynamics $\left({ }^{15 E} \mathrm{P}_{\mathrm{r}} \rightarrow{ }^{15 Z} \mathrm{P}_{\mathrm{g}}\right)$. (A) Sequential two-state model for the analysis (the ${ }^{15 E} \mathrm{P}_{\mathrm{r}} *$ spectrum was unresolved). (B) The extracted EADS from this analysis. Comparison of the EADS with the ${ }^{15 Z} \mathrm{Pg}_{\mathrm{g}}-{ }^{15 E} \operatorname{Pr}$ difference spectrum (unfilled circles) indicate that ${ }^{15 Z} \mathrm{Pg}_{\mathrm{g}}$ has not formed within the $200 \mu$ s window of this experiment. 


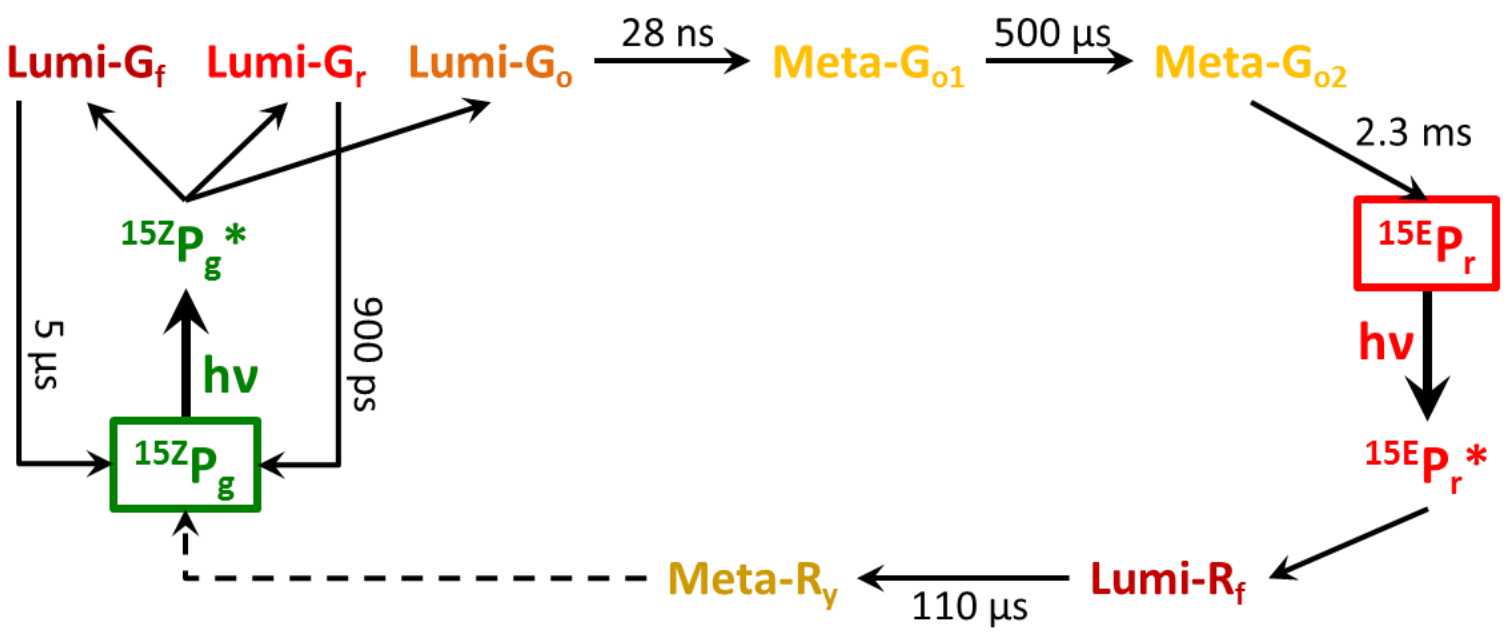

Figure 7. The proposed RcaE photocycle including both forward $\left({ }^{15 Z} \mathrm{P}_{\mathrm{g}} \rightarrow{ }^{15 E} \mathrm{P}_{\mathrm{r}}\right)$ and reverse $\left({ }^{15 E} \mathrm{P}_{\mathrm{r}} \rightarrow{ }^{15 Z} \mathrm{P}\right.$ ) reaction pathways. It is presumed that the protonation of PCB occurs at the final Meta- $\mathrm{G}_{02}$ to ${ }^{15 \mathrm{E}} \mathrm{P}_{\mathrm{r}}$ step in the forward reaction direction. It is unclear at what steps can be ascribed to the protonation and deprotonation reactions in the forward and reverse reaction directions, respectively; are not indicated in the scheme. 


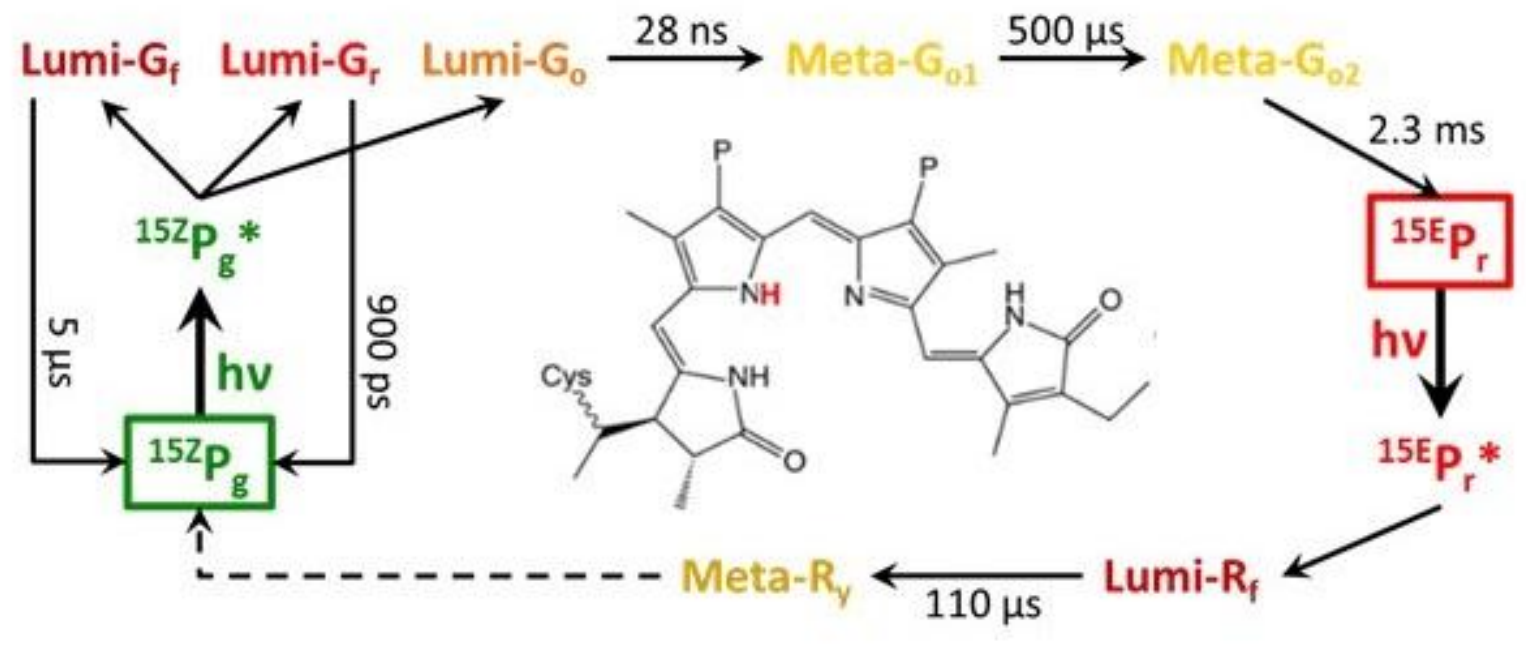

